Research Square

\title{
An Efficient Compression of Gray Scale Images using Wavelet Transform
}

\section{RAJIV RANJAN ( $\nabla$ rajivranjan.it@bitsindri.ac.in )}

Birsa Institute of Technology Sindri https://orcid.org/0000-0002-3408-7453

\section{Prabhat Kumar}

NIT Patna: National Institute of Technology Patna

\section{Research Article}

Keywords: Discrete Wavelet Transform, Huffman Coding, Image Compression, Quad tree Decomposition, Thresholding

Posted Date: July 6th, 2021

DOl: https://doi.org/10.21203/rs.3.rs-434952/v1

License: (1) This work is licensed under a Creative Commons Attribution 4.0 International License. Read Full License 


\title{
An efficient compression of gray scale images using Wavelet Transform
}

Rajiv Ranjan

Assistant Professor, BIT Sindri, Dhanbad, Jharkhand, India-828123

Email: rajivranjan.it@bitsindri.ac.in

ORCID ID: 0000-0002-3408-7453
Prabhat Kumar

Associate Professor, NIT Patna, Bihar, India- 800005

Email: prabhat@nitp.ac.in

\begin{abstract}
The rapid development of technology and the standardization of digital photography have led to an explosive growth in digital image distribution and reproduction. The enhancement of storage capacity in computer disks and advancement in networking have not been able to keep pace with the demands of handling, storing and finally transmitting huge volume of image data. Only proper image compression technologies seem to offer a solution to this challenge. The importance of digital image compression in multimedia applications has inspired extensive research all over the world. The present study recommends a newly formulated algorithm by computing Discrete Wavelet Transform (DWT) in combination with thresholding and quadtree decomposition. Findings prove that the proposed technique is at par with EZW image compression algorithm in terms of quality performance at the same bit rate, and obviates the need for employing any other conventional standard image compression techniques.
\end{abstract}

Keywords: Discrete Wavelet Transform, Huffman Coding, Image Compression, Quad tree Decomposition, Thresholding.

\section{Introduction}

The rapid change in the widespread use of computers, internet teleconferencing, and satellite communication have inspired recent researchers to focus their attention on digital image compression and maintain a standard quality for these multimedia applications. This enhances the need to promote resourceful and sophisticated techniques that would help to achieve optimal level of compression and fulfill the requirements of users. Compressing data [1] saves storage capacity; transfer files speedily and reduce the cost involved in storage hardware and network bandwidth.

Data redundancy is one of the fundamental components in digital image compression. Broadly speaking, three basic data redundancies have been identified as available in an image pixel viz. Coding redundancy, inter-pixel redundancy and psycho-visual redundancy. By applying appropriate encoding method, coding redundancy can be eliminated and information be represented in the form of codes. Inter-pixel redundancy is defined as failure to identify and utilize data relationships. It is of two kinds, namely, Inter-pixel spatial redundancy and Interpixel temporal redundancy. Inter-pixel spatial redundancy occurs due to a correlation between the neighboring pixels in an image. It depends upon the resolution of the image. Again, interpixel temporal redundancy is the statistical correlation between pixels from successive frames in video sequence. Conversely, Psycho-visual redundancies came into existence because the human 
perception of information in an image fails to receive quantitative analysis of every pixel or luminance value.

Image compression is achieved when any one or more of the above redundancies are reduced or eliminated. It comprises of Lossless Compression and Lossy Compression. The former explains compression in which the reconstructed image is an exact replica of the original image, with no information lost during the compression process. In contrast, lossy compression is just the reverse, where the reconstructed image is in no way exactly similar to the original one.

Image transforms are popularly adopted for decorating the pixels in image compression. To reduce the dependency between pixels, some standard image compression methods are employed. Few of these transformation tools include Discrete Fourier transform (DFT), Karhonen Loeve transform (KLT) [2], Principal component Analysis (PCA), Singular value decomposition (SVD), Discrete Cosine transform (DCT) [3,4], Discrete Wavelet transform (DWT) [5] and the like. Of all transformation tools available so far, DCT is most appropriately applied in one of the powerfully recognized JPEG [6] image compression standard. However, JPEG2000 [7] is based on DWT. Studies reveal that DWT has few advantages over DCT. Firstly, DCT is applied on block images and causes block artifacts, indicating loss of information. On the other hand, DWT provides much better compression ratio, without losing much information of image. This is because the latter does not work on blocks of images and its coefficient is localized.

Of late, one method of decomposing signal that has become increasingly popular is the use of wavelets. Entropy coding is administered on the DWT coefficients to compress the image and encourage efficient storage. The image is then passed through a series of analysis filter bank by using DWT. The analysis filter bank consists of Low-pass and High-pass filters that would allow both coarse and detail information to get extracted. Once image processing is over, image coefficients are divided into Approximation and Detail sub bands. To obtain the reconstructed image from these sub-bands, DWT uses synthesis filter bank.

Against this backdrop, the present authors have attempted to adopt a novel scheme by introducing DWT in combination with thresholding and quad tree decomposition to reduce symbol of coefficient for efficient compression of images. The result parameters of the proposed algorithm has been compared with Improved Embedded Zerotrees of wavelet transforms (IEZW) [8] based on the performance measure of peak signal-to-noise ratio (PSNR) at different low bitrate. Findings reveal impressive results for grayscale images. Moreover, it is easily comprehensible and manifests simple implementation on Matlab code. Thus, the proposed model attempts to establish an alternative measure to EZW without applying the concept of any other single conventional standard image compression methods.

This article has been divided into six sections. Section 1 is the introductory portion of the study. Section 2 deals with previous literature surveys related to the present study. Section 3 explains the preliminaries. In Section 4, the proposed algorithm is designated. Section 5 relates to the results obtained from experiments undertaken, while section 6 is the concluding part of the study.

\section{Related Work}

G. K. Wallace first proposed an Image compression standard known as JPEG algorithm [6] [9]. This tool is widely used for compression of gray scale images. It uses a lossy form of compression based on DCT. DCT is applied on $8 \times 8$ rectangular blocks of data. The signal is packed into few DCT coefficients after capturing the spatial redundancy by itself. It has a major 
drawback as it is subjected to block artifacts. Conversely, as discussed earlier in the introductory section, DWT is not based on block artifacts and its coefficient is more localized; thus indicating an advantage over DCT. Besides, the statistical qualities of the wavelet transform have been widely explored and today, the wavelet based image coding techniques have been considered as the latest, sophisticated and most useful development in the field of image compression. Studies reveal that the pyramids or dyadic wavelet decomposition [10-11] offers high energy compaction with high quality reconstructed images and is essentially useful in image compression [11-12]. For these reasons, within the past few years, DWT has become effectively operational for compression of the digital images [12-14] and has been suggested as a better alternative of DCT.

Of all image compression methods based on DWT approach known to us so far, embedded zero tree wavelet (EZW) [15] is the most popular. This newly proposed algorithm was first introduced by J.M. Shapiro in 1993. It is based primarily on four key concepts. Firstly, it should be a discrete wavelet transform (hierarchical sub-band decomposition). Secondly, it should predict the absence of significant information across scales by exploiting the self-similarity inherent in images; thirdly, it has entropy-coded successive-approximation quantization (SAQ), and finally, it enables to achieve universal lossless data compression via adaptive arithmetic coding. Nevertheless, it has few shortcomings. For example, some redundancy exists on few of the high frequency sub-bands. Here, for each SAQ iteration, the EZW coder scans all wavelet coefficients in each sub-band with respect to a given threshold. To overcome this problem, E.S. Kang [16] and others proposed a modified technique known as improved embedded zero tree wavelet coder (IEZW). Here, unlike the EZW coder, the proposed IEZW coder scans only coefficients of significant sub-bands and thereby significantly reduces the bit redundancy of the EZW. To eradicate this existing defect in EZW, J. Zhong designed another technique [17] based on quantized coefficient partitioning by using morphological operation. In this mechanism, instead of encoding the coefficients in each sub band line-by-line, regions in which most quantized coefficients were significant were extracted by morphological dilation and encoded first. This was followed by using zerotrees to encode the remaining space which had mostly zeros. Findings also confirmed that the proposed algorithm was far superior to the EZW. Also, the authors reported that obtained results were compared favorably with the most efficient wavelet-based image compression algorithms reported so far. In [18], A. Quafi et al proposed "A Modified Embedded Zero tree Wavelet (MEZW) Algorithm for Image Compression", where the authors modified Shapiro's EZW model of proposed algorithm. In their approach, the authors distributed entropy by using six symbols in place of four in EZW and also optimized the coding by a binary grouping of elements before coding. The results obtained showed remarkable improvement on the PSNR and compression ratio obtained by Shapiro, without affecting the computing time. Brahimi et al [19] proposed a technique for reducing the scanning and symbol redundancy of the existing EZW based on the use of six symbols instead of four. The main purpose of this technique was to avoid the encoding of the children of each significant coefficient based on the condition that there was no significant descendant. This enabled to [20] improve the quality of reconstructed image after decoding. In a newly formulated technique, Chen et al made use of the Compressed Sensing (CS) theory and EZW. Much later, extensive research on these areas prompted T. Brahimi et al [19] to propose a far better, effective technique. The method involved reducing the number of zerotrees as well as the scanning and symbol redundancy of the existing 
EZW, based on the use of a new significant symbol map which was represented in a more efficient way.

In this paper, to exploit the properties of the DWT, Quad tree decomposition and thresholding techniques have been developed in combination, as a new image compression algorithm which is expected to yield significantly better performance in the light of PSNR at the same bit-rate without using the zero - tree concept of EZW. Here, no single standard image compression methods like EBCOT [21], SPIHT [10] and JPEG 2000[7] have been employed. Moreover, the application of this method has been experimentally verified on different images and proved to provide promising results.

\section{Preliminaries}

In order to understand our proposed study better, it is essential to discuss the concepts behind the operational implementation of Discrete wavelet transform (DWT) and Quad tree decomposition methods in the field of data image compression. The former is applicable to decompose the image and reduce redundancy; whereas the latter is used to achieve high compression ratios and preserve edge integrity.

\subsection{Discrete wavelet transform}

DWT, known for its use as wavelet decomposition method, is used as a lossy coding compression. Its high energy compaction capability is expected to provide a well-designed coding technique that would reduce redundancy in an image and enable to achieve optimum compression. It works in a recursive manner and divides image into low pass and high pass elements.

The two-dimensional DWT is executed by a two-Channel wavelet filter bank. The image is initially scanned in horizontal direction, then passed through a filter to produce low pass and high pass frequency data. Once this output image data is generated, it is scanned in vertical direction to create various sub bands. The low frequency LL sub-band has significant information of the original image and is commonly called the Approximate Image; the LH, HL and HH sub-band denotes the Details of the Image. Each sub band is reduced to 1/4th the size of actual image [1] [22-24].

The basic underlying principal can be best understood by the mathematical equation and expression denoted below:

$f(x)=\sum_{k} c_{j 0}(k) \varphi_{j 0, k}+\sum_{j=j 0}^{\infty} \sum_{k} d_{j}(k) \Psi_{j, k}(x)$, where $c_{j 0}(k)$ and $d_{j}(k)$ are scaling and detail coefficient respectively.

Suppose function $f(x)$ is expanded and sequence of numbers of $f(x)$ discrete then resulting coefficient is recognized as the discrete wavelet transform. Most interestingly, DWT works perfectly well even when images are processed at multiple resolutions. 
The working approach of DWT is shown in Fig. 1. In this figure, output expressions are known as DWT coefficient. $h_{\varphi(-n)}$ and $h_{\Psi(-m)}$ are scaling and wavelet vectors which are low pass and high pass decomposition filters respectively. The output of Fig. 1 is calculated as:

$w_{\Psi}^{H}(j, m, n)=h_{\Psi}(-m) *\left[h_{\varphi}(-n) * w_{\varphi}(j+1, m, n) \mid n=2 k, k \geq 0\right] \mid m=2 k, k \geq 0$ Where * denotes convolution.

In Fig. 1, we get four lower scale components after input decomposition. $h_{\varphi(-n)}$ and $h_{\Psi(-m)}$ are called approximation coefficient and $w_{\phi}$ is created by these two. $\left\{w_{\Psi}^{i}\right.$ for $\left.i=H, V, D\right\}$ is known as detail coefficients.

The main application of DWT is in image compression where DWT is decomposing the images into lower and higher sub bands. Theoretically, though images can be decomposed up to the level of infinity, most researchers prefer to limit decomposition up to 3- level sub band [25-27].

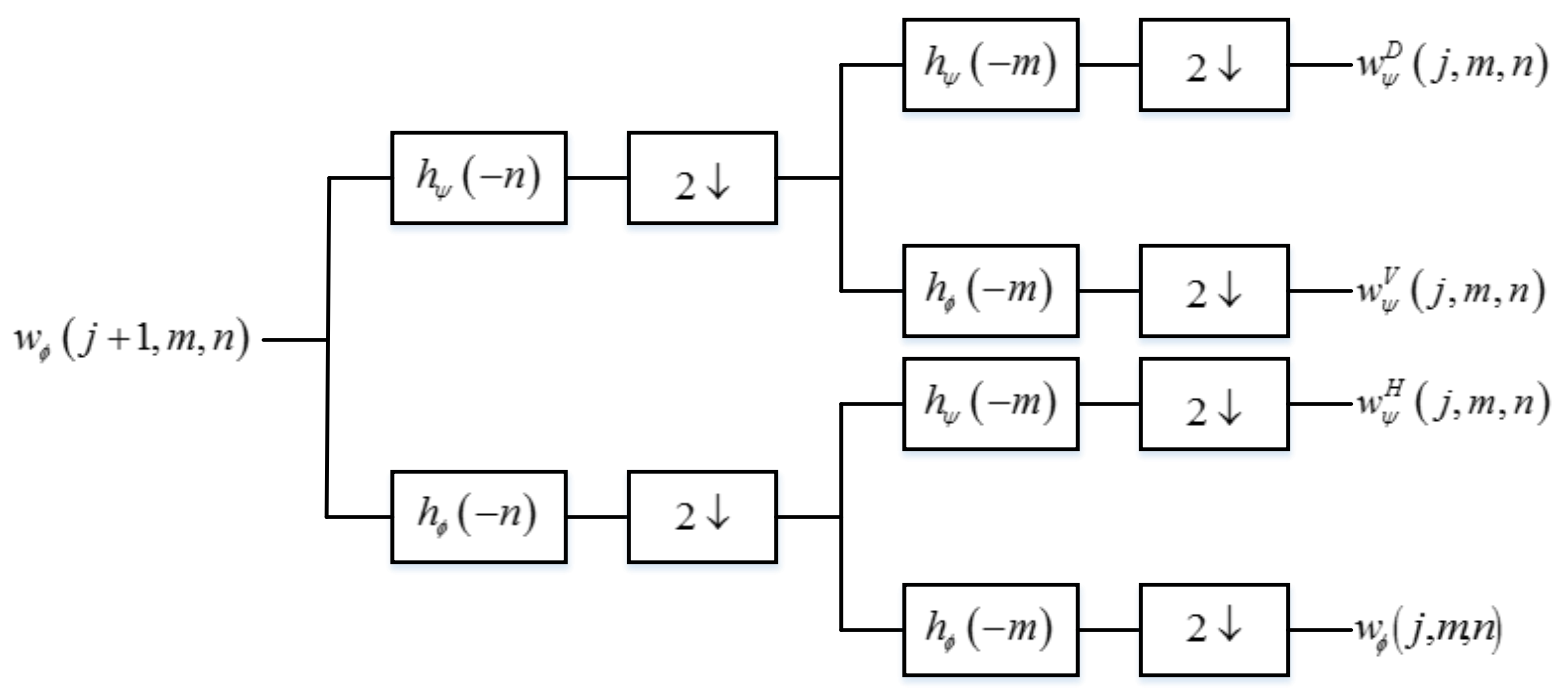

Fig. 1 DWT Decomposition

\subsection{Quadtrees}

A Quadtree is defined as a tree data structure in which each internal node has four children. Quadtrees are two-dimensional analog of octrees. They are generally used to partition a twodimensional space by recursively subdividing it into four quadrants or sections. The subdivided sections may be square or rectangular, or may have arbitrary shapes. Quadtree decomposition uses the qtdecomp function. This function works by dividing a square image into four equalsized square blocks first, and then testing each block to see if it meets some criterion of homogeneity (for example, if all the pixels in the block are within a specific dynamic range). In case a block meets the criterion; it is not divided any further. If it fails to meet the criterion, it is subdivided again into four blocks, and the test criterion is applied to those blocks. This process is repeated iteratively until each block meets the criterion. The result might have blocks of several different sizes [28]. 
Some common uses of quad trees are

- Image representation

- Image compression

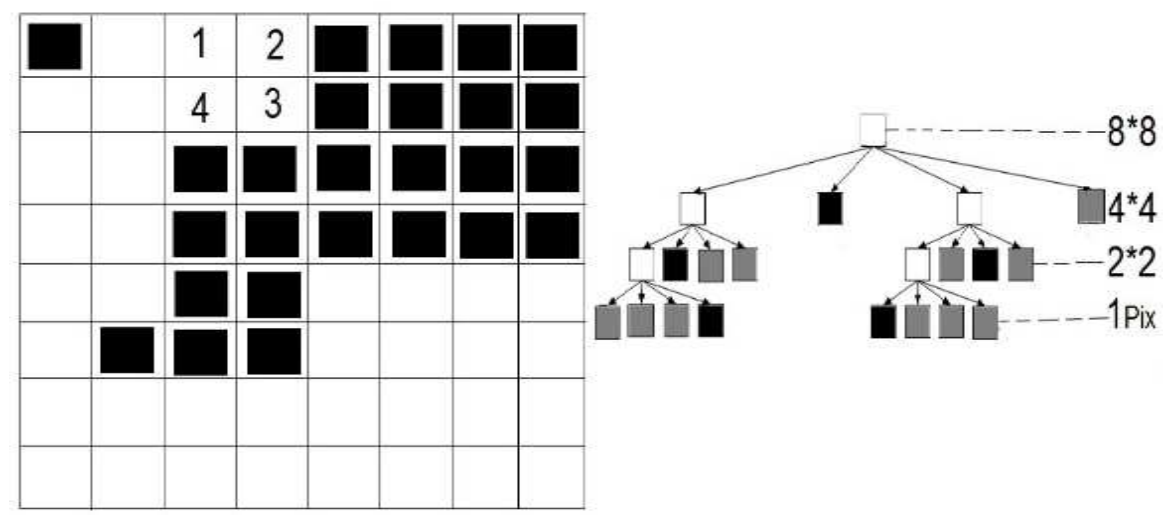

Fig. 2 Quad tree representation

4 Proposed Work

\subsection{Basic Approach}

The main objective of our approach is to use the features of DWT transformations. Firstly, DWT decomposed the key image after which Huffman Encoding was applied for additional improvement of the compression performance. The authors used a 2-level Haar wavelet transform for decomposing the 8-bit key images of size 512x512 pixels.

\subsection{Compression of DWT coefficients}

DWT details have zero mean and small variation. Using a Huffman Coding, DWT coefficients are restricted only to the most significant coefficients, ignoring the rest. The proposed algorithm is shown below:

Encoding Algorithm:

Input: A Gray Scale Image $I(X \times Y)$ of size $M \times N$

Output: A gray scale compressed reconstructed Image $I(X \times Y)$ of size $M \times N$ Algorithmic Steps:

Step 1: Apply DWT on the gray scale image, $I(X \times Y)$ so that it is decomposed into lower and higher sub bands.

Step 2: Obtain the median of the approximate coefficients, and use median as a base of log and calculate the logarithmic coefficients accordingly. The purpose of this step is to convert the 
higher value into lower value for enhancing the compression ratio. Preprocess the detail coefficients so that the detail coefficients are converted into the nearest integer.

Step 3: Apply entropy based smoothing on higher sub band according to their textual features to accept significant bits and discard the insignificant ones.

Step 4: Apply quadtree decomposition to reduce symbols of approximate and detail coefficients. Quadtree decomposition for approximate image is optional. It is used for higher bit-rate and lower PSNR value.

Step 5: Encode the approximate and details coefficients by Huffman coding.

Step 6: Get common compressed bit streams.

Step 7: Find Compression Ratio/bit-rate.

Decoding Algorithm

Input: Common compressed bit streams

Output: Reconstructed approximate image

Algorithmic steps:

Step1: Obtain compressed lower and higher sub band coefficient bit streams from common compressed bit streams.

Step2: Find reconstructed lower and higher sub band coefficient from compressed approximate and detail coefficient bit streams using reverse Huffman coding.

Step3: Other process is just the reverse of the encoding process.

Step4: To get reconstructed image using inverse DWT.

\subsection{Huffman Coding based Compression}

In our proposed method, a 3-level 'Haar Wavelet' transform decomposes the gray scale image. The next step involves getting approximate and detail Image. Next, the approximate and details coefficients are preprocessed. Once this operation is over, both coefficients are then encoded by Huffman code to get a sequence of digital data. To get the reconstructed image, the binary data is decoded. For this, the encoding process is just reversed. The concept behind this newly designed method is best illustrated by the algorithm and flowchart as given below: 


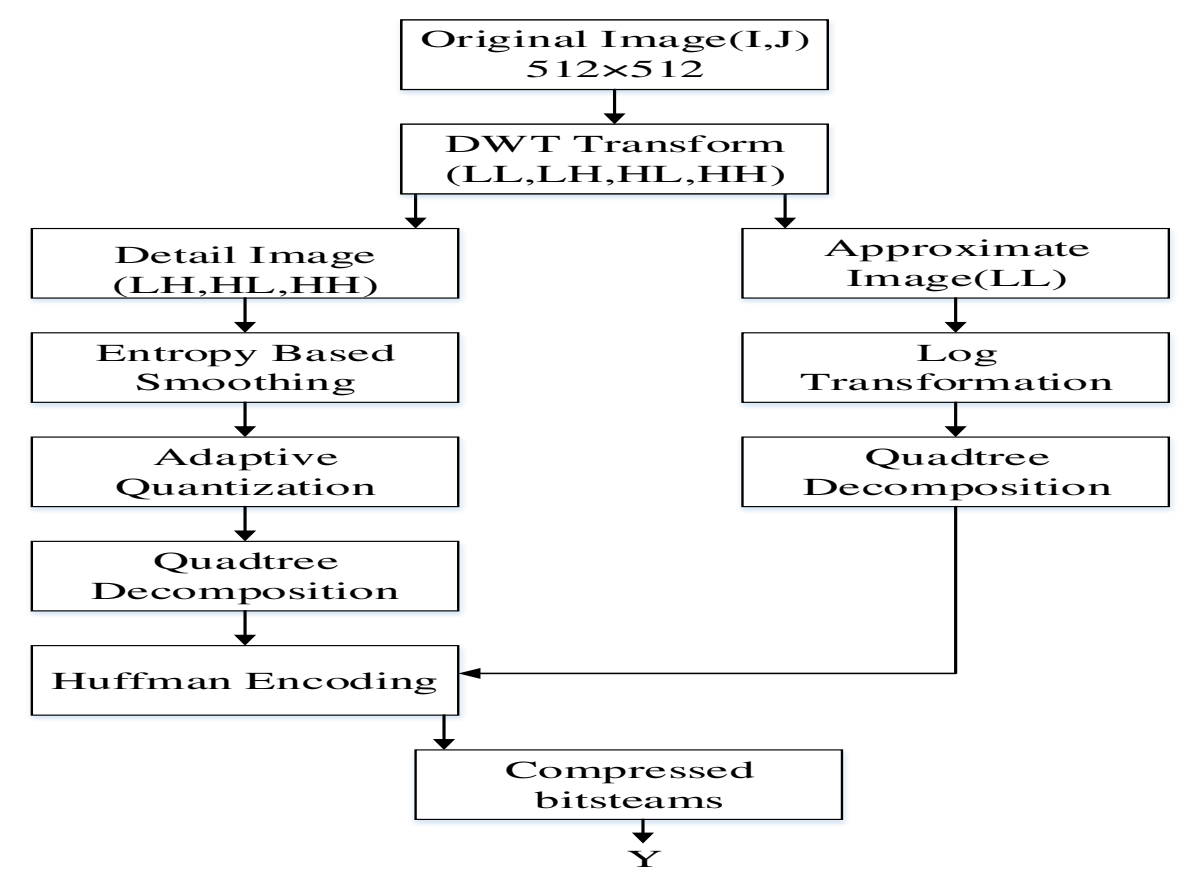

Fig. 3 Overall scheme of proposed image compression algorithm

5 Experimental Results and Discussions

\subsection{Performance Evaluation}

The performance of lossy compression approaches can be estimated with the help of certain indicators mentioned below.

a. Peak-Signal-to-noise-ratio (psnr): psnr is the usual scale to compute the compressed image worth. For the general case of 8 bits per picture element of key image, the peak snr (psnr) can be expressed as [29-30]

$\operatorname{psnr}(d B)=10 \log _{10}\left(\frac{255^{2}}{m s e}\right)$

Where worth 255 is utmost possible worth that can be attained by the image signal. mse in (1) denotes the mean squared error of the image expressed as $m s e=\frac{1}{n} \sum_{i} \sum_{j}(f(i, j)-F(i, j))^{2}$

Here $\mathrm{n}$ is the whole quantity of pixels,

$F(i, j)$ indicates the pixel value in the compressed image and $f(i, j)$ expresses a pixel value in the original image.

b. Compression Competence(CR):Compression ratio[31] is a scale to measure compression competence, which is defined Mathematically as follows:

$$
C R=\frac{S_{\text {original }}}{S_{\text {compressed }}}
$$


Where $S_{\text {original }}$ the dimension of the original is image data and $S_{\text {compressed }}$ is the dimension (numeral of bits) of the compressed image data.

Bit-per-pixel $(\mathrm{BPP})=8 / \mathrm{CR}$. For grayscale image .

c. Structure similarity index (SSIM): For evaluating the excellence of a reconstructed image, SSIM [32] is one of the factors.

The image compression algorithms proposed in this paper were applied on different grayscale images. Fig. [5(a-f)] represents the results on test image 'Lena'. The performance of our proposed algorithm applied on different set of test images 'Airplane', 'Lena', 'Peppers', 'Barbara', 'Goldhill' and 'Sailboat' of size 512×512 are represented in Fig. 4.

\subsection{Performance Evaluation of Proposed Image Compression Procedure}

The performance of the proposed Image Compression method has been analyzed using such parameters as PSNR (Peak Signal to Noise Ratio), SSI (Structural Similarity Index), CR (Compression Ratio) and BPP (Bit-per-pixel). In order to ensure consistency, the same test images used in [9] were employed. (Bold values indicate the best performances obtained in terms of PSNR (dB)).

Table 1 confirms that the obtained results are very close to those obtained by IMP1EZW [8]; without using any of the standard image compression algorithm such as EZW, SPIHT, EBCOT and JPEG2000. The Haar wavelet has been opted for decomposing the images using DWT.

From Fig. 6, the authors have noticed some constructive improvement on images, and its quality as shown in Fig. 5(a)-(f).The program has been established in Matlab. The results obtained are presented in Table 1. As expected, the outcome of the proposed method proved more promising than that of IMPIEZW at most of the bit-rates within a given range for all test images. Fig. 7 shows the view of images at different stages of proposed method. 
Table 1. Comparative analysis of PSNR of Reconstructed images.

\begin{tabular}{|c|c|c|c|}
\hline Image & $\begin{array}{c}\text { Bitrate } \\
\text { (bpp) }\end{array}$ & IMPIEZW[8] & $\begin{array}{l}\text { Proposed } \\
\text { (DWT) }\end{array}$ \\
\hline \multirow{6}{*}{ Airplane } & 0.03125 & 23.8106 & 22.3540 \\
\hline & 0.0625 & 25.7746 & 25.7853 \\
\hline & 0.125 & 28.1217 & 27.0395 \\
\hline & 0.25 & 31.2195 & 29.6331 \\
\hline & 0.5 & 34.9951 & 32.7346 \\
\hline & 1 & 40.3689 & 34.5345 \\
\hline \multirow{6}{*}{ Lena } & 0.03125 & 23.8158 & 22.8452 \\
\hline & 0.0625 & 26.2563 & 25.7797 \\
\hline & 0.125 & 28.9792 & 29.0153 \\
\hline & 0.25 & 31.6442 & $\mathbf{3 1 . 5 7 7 2}$ \\
\hline & 0.5 & 34.7579 & 34.2418 \\
\hline & 1 & 38.6311 & 35.6323 \\
\hline \multirow{6}{*}{ Peppers } & 0.03125 & 24.5045 & 23.0027 \\
\hline & 0.0625 & 27.0365 & 27.9992 \\
\hline & 0.125 & 29.8714 & 28.6553 \\
\hline & 0.25 & 33.0125 & 29.9554 \\
\hline & 0.5 & 35.7291 & 31.0221 \\
\hline & 1 & 38.3256 & 32.4194 \\
\hline \multirow{6}{*}{ Barbara } & 0.03125 & 21.1572 & 21.3986 \\
\hline & 0.0625 & 22.4375 & 23.8611 \\
\hline & 0.125 & 23.4877 & 23.9054 \\
\hline & 0.25 & 25.925 & 24.0173 \\
\hline & 0.5 & 29.0292 & 26.0830 \\
\hline & 1 & 33.8263 & 29.2387 \\
\hline \multirow{6}{*}{ Goldhill } & 0.03125 & 23.5271 & 24.2379 \\
\hline & 0.0625 & 24.4855 & 27.1712 \\
\hline & 0.125 & 26.9261 & 27.3161 \\
\hline & 0.25 & 28.7573 & 28.4243 \\
\hline & 0.5 & 31.1567 & 29.4883 \\
\hline & 1 & 34.4445 & 31.3273 \\
\hline \multirow{6}{*}{ Boat } & 0.03125 & 22.5333 & 21.9817 \\
\hline & 0.0625 & 24.6006 & 22.4062 \\
\hline & 0.125 & 26.3312 & 26.1665 \\
\hline & 0.25 & 28.7099 & 27.0335 \\
\hline & 0.5 & 31.9414 & 29.9632 \\
\hline & 1 & 36.0863 & 31.0084 \\
\hline
\end{tabular}




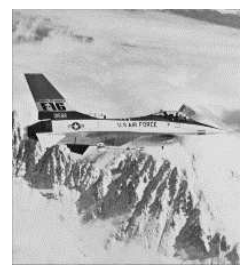

(a)Airplane

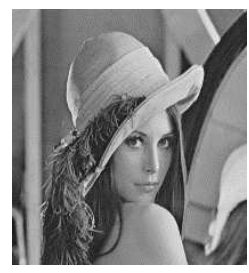

(b) Lean

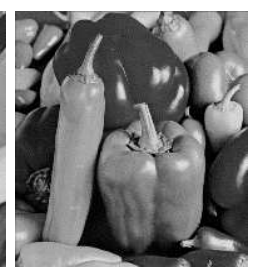

(c) Pepper

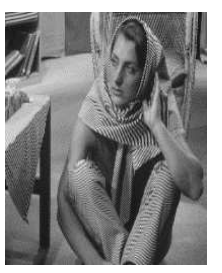

(d) Barbara

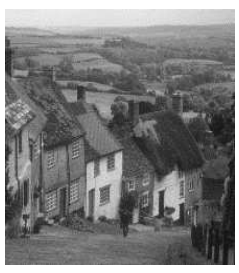

(e) Goldhill

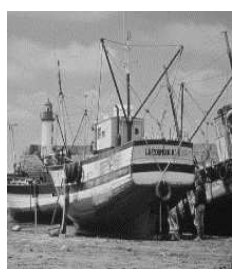

(f) Boat

Fig. 4 Grayscale test images of size $512 \times 512$
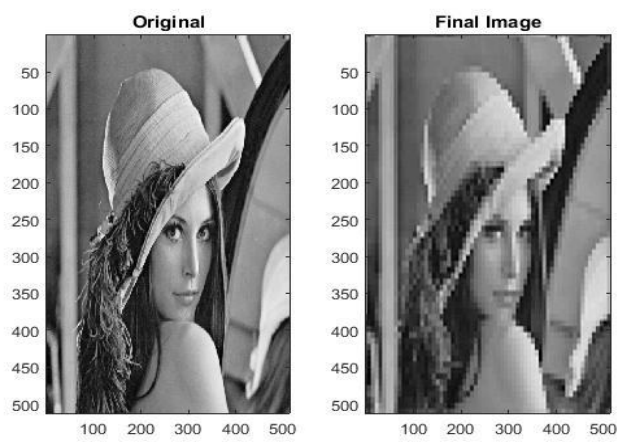

(a)

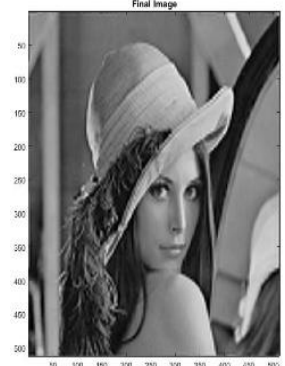

(c)

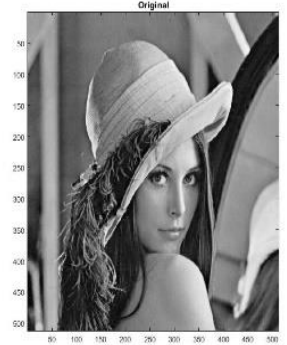

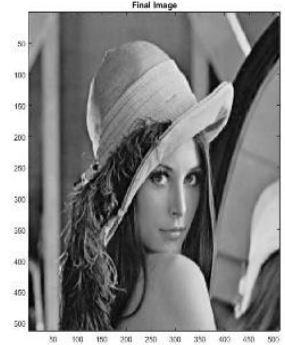

(e)
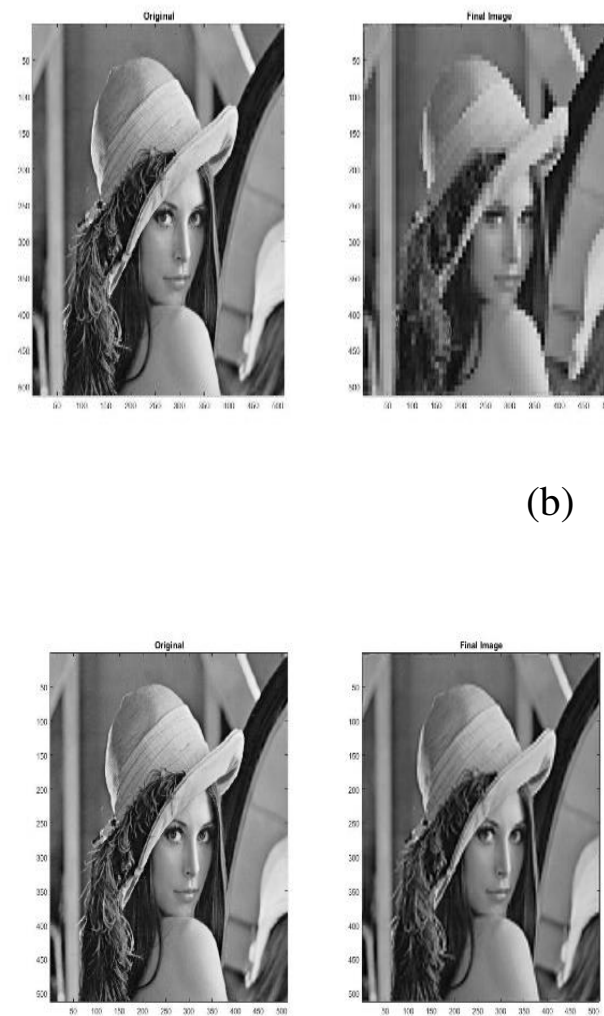

(d)
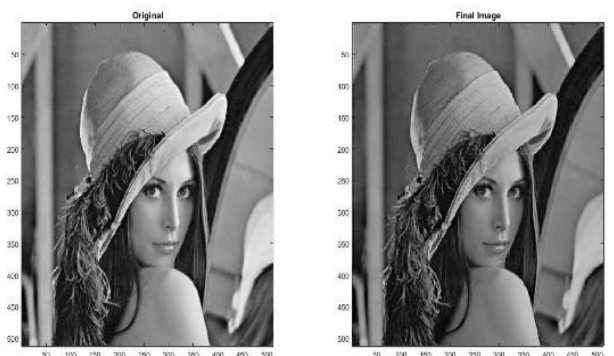

(f)

Fig. 5 Lena (Fig a-f; reconstructed image) at different bit-rates $0.03125,0.0625,0.125,0.25,0.5$ ,1 with PSNR 22.9172, 25.8127, 29.0219, 31.5810,34.3612,35.6233 and SSIM $0.7013,0.7436,0.8133,0.8519,0.9023,0.9117$ respectively. 


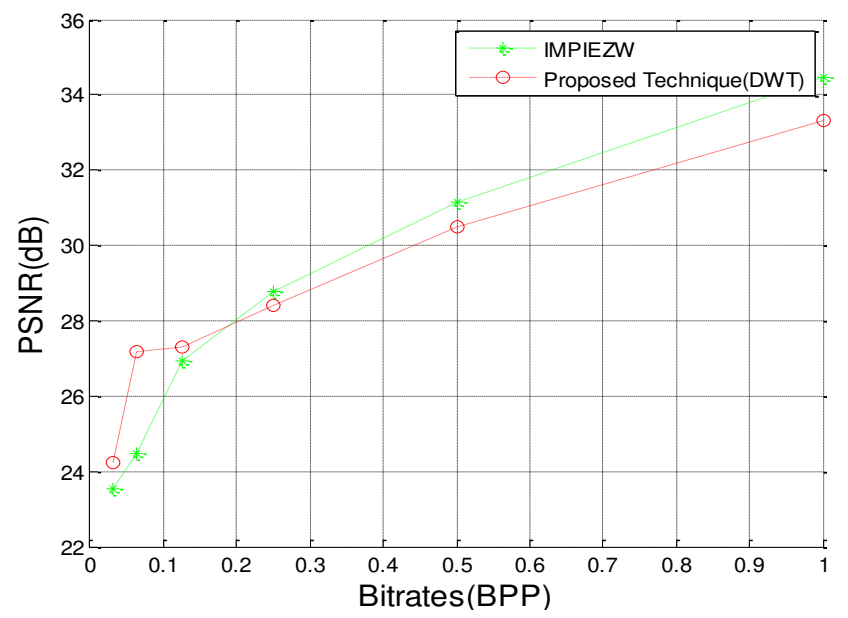

Fig. 6 Performance comparisons of Proposed Coders (DWT) against the methods IMPIEZW [8] for Gold-hill gray scale image at the same range of bitrates [0.03125 0.06250 .1250 .250 .51 ] (BPP).

\section{Conclusion}

In the present paper, the authors presented a technique where the image has been decomposed by the combined application of DWT and Haar wavelet transform. The said method has divided image into two parts. One is the approximate image, playing a key role since approximate coefficients possess one of the most sensitive data in DWT transform. As discussed earlier, approximate image is a compressed representation of the original one and has therefore been handled here with utmost care; else, any impact upon the reconstructed image is expected to greatly reduce the PSNR value. The second part includes the stage of preprocessed image where the detailed coefficients are smoothed based on their textual feature and their outcomes are quantized. This causes reduction in the number of insignificant symbols. The quadtree decomposition functions in order to significantly reduce the data size and allow working on smaller data. The outcomes of the proposed technique have been compared with various stateof-the-art image compression techniques. The quantitative and visual results showed the superiority of the proposed algorithm over the state-of-the-art techniques.

\section{Declarations:}

\section{Funding: Nil}

Conflict of interest: The authors declare that they have no conflict of interest.

Availability of data material: Not applicable

Code availability: Not applicable

\section{References}

1. Ranjan, R. (2021) "Canonical Huffman Coding Based Image Compression using Wavelet", Wireless Personal Communications., 117(3):2193-2206.

2. Leung, Raymond, and David Taubman. (2005) "Transform and embedded coding techniques for Maximum efficiency and random accessibility in 3-D scalable compression." IEEE Transactions on Image Processing, 
14(10): 1632-1646.https://doi.org/10.1109/TIP.2005.851707

3. Song, Han Sae, and Nam Ik Cho. (2009) "DCT-based embedded image compression with a new coefficient sorting method." IEEE Signal Processing Letters, 16(5): 410-413.https://doi.org/10.1109/LSP.2009.2016010

4. Ponomarenko, Nikolay N., et al. (2007) "High-quality DCT-based image compression using partition schemes." IEEE Signal Processing Letters, 14(2): 105-108.https://doi.org/10.1109/LSP.2006.879861

5. Song, Xiaoying, et al. (2016) "Three-dimensional separate descendant-based SPIHT algorithm for fast compression of high-resolution medical image sequences." IET Image Processing, 11(1):80-87.https://doi.org/10.1049/ ietipr.2016.0564

6. Wallace, Gregory K. (1992) "The JPEG still picture compression standard." IEEE transactions on consumer electronics, 38(1): 18- 34.

7. Christopoulos, Charilaos, Athanassios Skodras,and Touradj Ebrahimi. (2000) "The JPEG2000 still image coding system: an overview." IEEE transactions on consumer electronics 46(4): 1103-1127. https://doi.org/10.1109/30.920468

8. T. Brahimi,F. Laouir,Larbi Boubchir,Arab Ali-Cherif, (2017) "An improved wavelet-based image coder for embedded greyscale and colour image compression”, Int. J. Electron. Commun.(AEU) 73:183-192.

9. G. K. Wallace. (1990) "Overview of the JPEG (ISO/CCITT) still image compression standard. Image Processing Algorithms and Techniques", Proceedings of the SPIE, 1244: 220-233.

10. A. Said, W.A. Pearlman (1996) "A new fast and efficient image codec based on set partitioning in hierarchical trees", IEEE Trans. Circ. Syst. Video Technol. 6 (3):243-250.

11. T. Brahimi, A. Melit, F. Khelifi and D. Boutana, (2006) "Improvements to SPIHT for lossless image coding," in Proc. Of ICTTA'06, pp. 1445-1450.

12. S. Mallat,( 1989) "A theory for multiresolution signal decomposition: the R. wavelet representation, "IEEE Trans. Pattern Analysis and Machine Intelligence, 11(7).

13. M. Antonini, M. Barlaud, P. Mathieu, and I. Daubechies ,( 1992) "Image coding using wavelet transform, " IEEE Trans. Image Processing, 1(2):205-220.

14. S.Welstead, (1999) " Fractal and wavelet image compression techniques, "Spie Optical Engineering Press, Washington, USA.

15. J.M. Shapiro, (1993) Embedded image coding using zero trees of wavelet coefficients, IEEE Trans. Signal Process. 41(12): 3445-3462.

16. Kang ES, Tanaka T, Ko SJ. (1999) Improved embedded zerotree wavelet coder. Proc IEE Electron Lett; 35(9):705-6.

17. C. Cai, S.K. Mitra, Runtao Ding, (2002) "Smart wavelet image coding: X-tree approach, Signal Processing", $82: 239-249$.

18. J.Zhong, C.H. Leung, (2000) "An Improved Embedded Zerotree wavelet Image coding method based on coefficient partitioning using Morphological operation, International Journal of Pattern Recognition and Artificial Intelligence”, 14(6): 795-807.

19. A. Ouafi, A. T. Ahmed, Z. Baarir, A. Zitouni, (2008) "A Modified Embedded Zerotree Wavelet (MEZW) Algorithm for Image Compression”, J Math Imaging, 30: 298-307

20. T. Brahimi,F. Laouir, N. Kechacha, An efficient wavelet-Based Image Coder,IEEE Conf.,2008,pp.1-4

21. Z. Chen, C. Mu, (2014) "An Improvement of Embedded Zerotree wavelet coding Based on Compressed Sensing", IEEE Conf.

22. D. Taubman, (2000) High performance scalable image compression with EBCOT, IEEE Trans. Image Process, 9(7):1158-1170.

23. Michail Krinidis,Nikos Nikolaidis, Ioannis Pitas, (2007) "The discrete modal transform and its application to lossy image compression”, Signal Processing: Image Communication, 22:480-504.

24. ISO/IEC10918-1 ITU-TRec.T.81, Information Technology - Digital Compression and Coding of ContinuousTone Still Images, 1992.

25. ISO/IEC15444-1 j ITU- TRec.T.800, Information Technology-JPEG 2000 Image Coding System: Core Coding System, 2002.

26. S. Benchikh, and M. Corinthios, (2011) "A Hybrid Image Compression Technique Based on DWT and DCT Transforms", Advanced Infocom Technology 2011 (ICAIT 2011), IEEE, 2011, pp. 1-8.

27. Tim Braylants, Adrian Munteanu, Peter Schelkens, (2015) "Wavelet based volumetric medical image compression", Signal Processing: Image Communication, 31:112-133.

28. F. Keissarian, "A New Quadtree-based Image Compression Technique using Pattern Matching Algorithm”,ICCES,12(4): 137-143

29. Cosman, P.C., Gray, R.M. and Olshen,R.A., (1994) "Evaluating quality of compressed medical images: SNR, 
Subjective rating, and diagnostic accuracy. Proceedings of the IEEE, 30: 857-865.

30. R.Reddy M,R.KS,V.B,S.SD, (2018) "A new approach for the image compression to the medical images using PCA-SPIHT”,Biomedical Research ,Special Issue:S481-S486,ISSN 0970-938X

31. O. Yildirim,R.S. Tan and U.R. Acharya,(2018) An efficient compression of ECG Signals using deep convolutional autoencoders, Elsevier, Cognitive System Research52(2018) 198-211.

32. P.M. Latha,A.A. (2018) Fathima,Collective compression of Images using Averaging and Transform Coding, Elsevier, Measurement, S0263-2241(18): 31181-3.
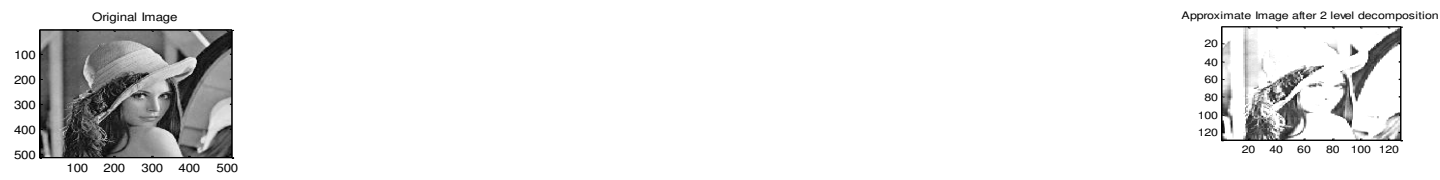

Horizontal Detail Image after EBS

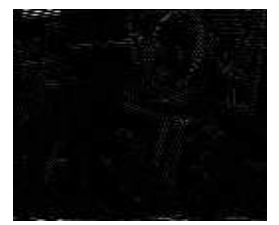

Horizontal Detail Image after Thresholding

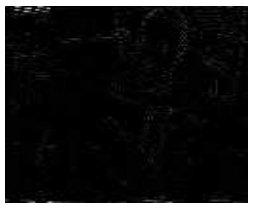

Horizontal Detail Image after Quantization

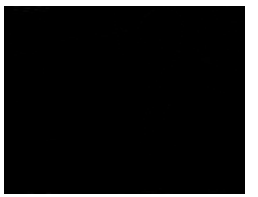

Vertical Detail Image after EBS

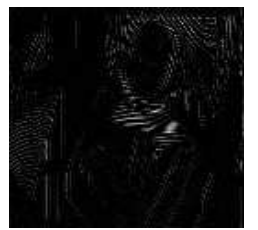

Vertical Detail Image after Thresholding

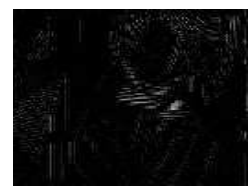

Vertical Detail Image after Quantization
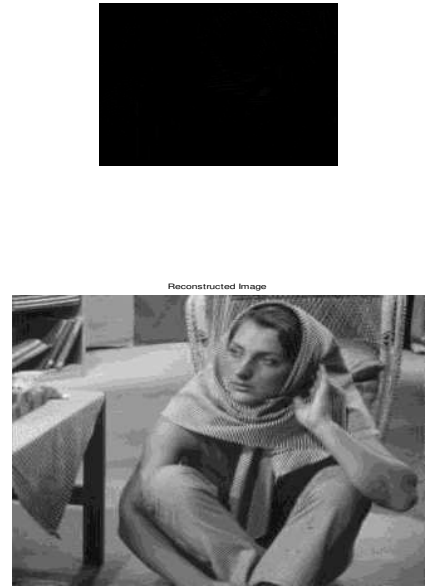

Diagonal Detail Image after EBS

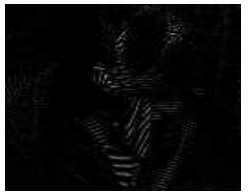

Diagonal Detail Image after Thresholding

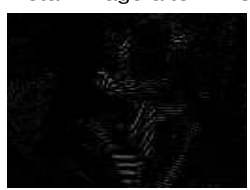

Diagonal Detail Image after Quantization

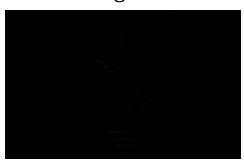

Fig. 7 Demonstration of Barbara image at bit-rate 0.0625 (BPP) at different stages of proposed Method 